\title{
RELATIVISTIC QUANTUM MECHANICS AND THE BOHMIAN INTERPRETATION
}

\author{
Hrvoje Nikolić
}

Theoretical Physics Division

Rudjer Bošković Institute

P.O.B. 180, HR-10002 Zagreb, Croatia

E-mail: hrvoje@thphys.irb.hr

\begin{abstract}
Conventional relativistic quantum mechanics, based on the Klein-Gordon equation, does not possess a natural probabilistic interpretation in configuration space. The Bohmian interpretation, in which probabilities play a secondary role, provides a viable interpretation of relativistic quantum mechanics. We formulate the Bohmian interpretation of many-particle wave functions in a Lorentz-covariant way. In contrast with the nonrelativistic case, the relativistic Bohmian interpretation may lead to measurable predictions on particle positions even when the conventional interpretation does not lead to such predictions.
\end{abstract}

Key words: relativistic quantum mechanics, Klein-Gordon equation, Bohmian interpretation.

\section{INTRODUCTION}

It is well known that the probabilistic interpretation of the nonrelativistic Schrödinger equation for particles without spin does not work for its relativistic generalization - the Klein-Gordon equation (see, e.g., Ref. [1]). This is related to the fact that the Klein-Gordon equation

$$
\left(\partial^{\mu} \partial_{\mu}+m^{2}\right) \psi(x)=0
$$

(where $x=(t, \mathbf{x})$ and we take $\hbar=c=1$ ) contains a second time derivative, instead of the first time derivative that appears in the Schrödinger equation. The quantity $|\psi(x)|^{2}$ cannot be interpreted as a probability density for a particle to have the position $\mathbf{x}$ at time $t$ because then the total probability $\int d^{3} x|\psi(x)|^{2}$ would not be conserved in time. One can introduce the conserved current

$$
j^{\mu}=i \psi^{*} \stackrel{\leftrightarrow}{\partial^{\mu}} \psi
$$

(where $a \stackrel{\leftrightarrow}{\partial^{\mu}} b \equiv a \partial^{\mu} b-b \partial^{\mu} a$ ), but the time component $j^{0}(x)$ cannot be interpreted as a probability density because it is not positive definite. Note that this is not a problem for the scattering formalism where one assumes that wave functions are positive-frequency plane waves asymptotically. However, quantum theory is more than a theory of scattering and the problem of negative $j^{0}$ arises outside the scattering regime.

The usual solution of this problem consists in adopting the second quantization of $\psi$ (see, e.g., Ref. [2]), where $\psi$ is not a wave function determining probabilities, but an observable (called field) that satisfies the quantum uncertainty laws. However, if, at the fundamental level, $\psi$ should not be interpreted as a wave function that determines probabilities of particle positions, then it 
is not clear at all why such an interpretation of $\psi$ is in such good agreement with experiments for nonrelativistic particles.

Using the Bohmian interpretation [3, 4, 5, 6, 7, and the theory of particle currents [8, 9, 10, a theory that consistently combines the postulates of second quantization (relativistic quantum field theory) with the postulates of first quantization (quantum mechanics) has recently been proposed in Refs. 11, 12]. The comparative advantages of the Bohmian interpretation over other interpretations applied to relativistic quantum mechanics have been discussed in Ref. [13]. In Refs. 11, 12, the equations that determine the Bohmian trajectories of relativistic quantum particles described by many-particle wave functions were written in a form that required a preferred time coordinate. Indeed, it is often argued that any relativistic hidden variable theory compatible with quantum nonlocality must introduce a preferred Lorentz frame. However, as demonstrated in Ref. 14, this is not necessarily so. In the present paper, we further elaborate some of the ideas introduced in Refs. [1] and [14] to formulate a fully Lorentz-covariant Bohmian interpretation of relativistic quantum mechanics for particles without spin. (The generalization to other spins is straightforward.)

As in Refs. 14, 11, it appears that particles may be superluminal, i.e., faster than light in the vacuum. Before discussing how this happens in the framework of the relativistic Bohmian interpretation, it is important to emphasize that, contrary to frequent claims, the principle of Lorentz covariance does not forbid superluminal velocities and superluminal velocities do not lead to causal paradoxes (see, e.g., Refs. [15, 16]). Indeed, there is large evidence that various relativistic interactions may cause ordinary particles or waves to propagate superluminally [17. 18, 19, 20, 21, 22, 23, 24.

As noted in Ref. [14, the Lorentz-covariant Bohmian interpretation of the many-particle Klein-Gordon equation is not statistically transparent, i.e., the statistical distribution of particle positions cannot be calculated in a simple way from the wave function alone without the knowledge of particle trajectories. This lack of statistical transparency is one of the main objects of the present paper, the physical meaning of which is qualitatively discussed in Sec. 2 The quanitative formulation of the Lorentz-covariant Bohmian interpretation is given in Sec. 3, while some statistical predictions are discussed in Sec. 4. The conclusions are drawn in Sec. 5.

\section{STATISTICAL TRANSPARENCY}

Consider a dynamical theory of configuration variables that may or may not involve the existence of trajectories of configuration variables. We say that this theory is statistically transparent if one can calculate the probabilites for possible outcomes of measurements of configuration variables in a natural way that, in particular, does not refer to any information on the (possibly existing) trajectories. A remarkable property of nonrelativistic quantum mechanics (QM) is that it $i s$ statistically transparent. In other words, one can calculate the probability density for particle positions directly from the wave function, without knowing particle trajectories. Therefore, from the practical calculational point of view, the concept of a particle trajectory in nonrelativistic QM is simply superfluous. This is certainly the main reason that the Bohmian interpretation of nonrelativistic QM is ignored by most physicists, and is probably the main reason for the wide belief that, in nonrelativistic QM, particle trajectories simply do not exist. In other words, statistical transparency is the main reason for the wide belief that QM is a fundamentally probabilistic theory. Without statistical transparency, there would no longer be a good reason for such a belief.

However, nonrelativistic QM is certainly not the most fundamental theory that we know. Is statistical transparency a fundamental principle, or just a property of some approximative theo- 
ries? For example, what about relativistic (i.e., Lorentz-covariant) theories without a preferred time? The following intentionally chosen facts suggest that statistical transparency may not be a fundamental principle of nature:

1. Classical mechanics, either nonrelativistic or relativistic, is not statistically transparent.

2. Relativistic quantum mechanics based on the Klein-Gordon equation or some of its generalizations is not statistically transparent (owing to the reasons discussed in Sec. 1).

3. The relativistic Bohmian interpretation of the Dirac equation is statistically transparent, but the corresponding many-particle relativistic generalization is not statistically transparent [14] (unless a preferred time coordinate is determined in a yet unknown dynamical way [25]).

4. Nonrelativistic QM is statistically transparent, but it is not completely statistically transparent, in the sense that, for a fixed time $t$, it gives the probability density $\rho\left(x^{1}, x^{2}, x^{3}\right)$, but, for example, for a fixed $x^{3}$, it does not give a probability density $\rho\left(x^{1}, x^{2}, t\right)$.

5. The background-independent quantum gravity based on the Wheeler-DeWitt equation lacks the notion of time and is not statistically transparent [26, 27].

Note that, given fact 4, one should not be surprised with the fact that relativistic QM may not be statistically tranparent. Since time and space should play equal roles in a relativistic theory, from fact 4 one might expect that relativistic QM should be either completely statistically transparent or not statistically transparent at all. For the Klein-Gordon equation, it is the latter possibility that actually realizes.

The lack of statistical transparency does not automatically imply that the probabilities cannot be calculated at all. For example, in classical mechanics, if we know the probability distribution $\rho(\mathbf{x})$ at some initial time and the exact particle velocity for each possible initial position $\mathbf{x}$, we can calculate $\rho(\mathbf{x})$ at any other time by calculating particle trajectories for all possible initial positions. (For practical purposes, it is usually sufficient to calculate the trajectories for a large but finite sample of initial positions.) In a similar way, in principle, one can obtain statistical predictions from any quantum theory provided that deterministic trajectories do exist. On the other hand, if deterministic trajectories do not exist, then it is not clear at all how to assign any physical interpretation to a quantum theory that lacks statistical transparency. Sinse most of the interpretations of QM do not incorporate deterministic trajectories, we conclude that, in general, the Bohmian interpretation of quantum theory is more powerful than most other interpretations (see also Ref. [13]).

The lack of statistical transparency in relativistic QM translates into the lack of statistical transparency in relativistic Bohmian mechanics. This feature is often considered as a drawback of Bohmian mechanics, which motivates investigations of various modifications of the formalism (e.g., based on the introduction of a preferred time coordinate [28] or on the representation of the Klein-Gordon equation by a first-order differential equation [29]), such that statistical transparency is recovered. Such modifications typically imply statistical transparency even without the Bohmian interpretation, making the Bohmian interpretation unnecessary for practical predictions.

In our view, the lack of statistical transparency is not a drawback of a Bohmian theory, but rather its virtue. The reason is the following. The statistical predictions of nonrelativistic Bohmian mechanics are equivalent to those of the conventional interpretation, which makes the scientific value of the Bohmian interpretation questionable, because its basic assumption the existence of particle trajectories - cannot be verified experimentally. On the other hand, 
for a quantum theory without statistical transparency, the statistical predictions of a Bohmian interpretation are not equivalent to those of the conventional interpretation, simply because, in general, the conventional interpretation does not provide any statistical predictions for configuration variables at all. This opens the possibility of verifying the validity of a Bohmian interpretation experimentally, i.e., of obtaining some statistical predictions by explicitly calculating the trajectories and comparing the predictions with experiments. If the predictions turn out to disagree with experiments, then one may conclude that this particular Bohmian theory is wrong. (One of the main criteria for a meaningful scientific theory is that it can be falsified.) If the predictions turn out to agree with experiments, then one can conclude that the theory is correct and that the particle trajectories are very likely to really exist (instead of merely being a calculational tool [30]), because the same predictions cannot be obtained without the trajectories.

In Bohmian mechanics without statistical transparency, one can obtain statistical predictions at later times if the probability distribution $\rho(\mathbf{x})$ is known at the initial time. However, how to know this initial $\rho$ ? Since the theory is not statistically transparent, in general, there is no way of knowing it in a purely theoretical way. Fortunately, in some special cases, it is possible to know the initial $\rho$ in a purely theoretical way, even without statistical transparency at all times. For example, assume that particles are slow initially, so that the nonrelativistic approximation can be used. Then one can invoke either the nonrelativistic typicality argument 31, 32] or the nonrelativistic quantum $\mathrm{H}$-theorem [33] to conclude that the quantum equilibrium takes place initially, i.e., that the initial probability distribution is given by $\rho=\psi^{*} \psi$. Then, assume that later interactions are such that particles become relativistic, so that $\psi$ is a relativistic solution which is not statistically transparent (i.e., the quantity $j^{0}$ is not positive definite) at later times. In such a case, the statistical predictions for particle positions at later times can be obtained with the Bohmian interpretation, but not with the conventional interpretation. Such predictions can be compared with experiments. This motivates us to study the relativistic Bohmian interpretation at the quantiative level, which we do in the subsequent sections.

\section{LORENTZ-COVARIANT BOHMIAN MECHANICS}

Let $\hat{\phi}(x)$ be a scalar field operator satisfying the Klein-Gordon equation (11). For simplicity, we take $\hat{\phi}$ to be a hermitian uncharged field, so that the negative values of the time component of the current (to be defined below) cannot be interpreted as negative-charge densities. Let $|0\rangle$ be the vacuum and $|n\rangle$ an arbitrary $n$-particle state. These states are Lorentz-invariant objects. The corresponding $n$-particle wave function is [34, 11 .

$$
\psi\left(x_{1}, \ldots, x_{n}\right)=(n !)^{-1 / 2} S_{\left\{x_{a}\right\}}\left\langle 0\left|\hat{\phi}\left(x_{1}\right) \cdots \hat{\phi}\left(x_{n}\right)\right| n\right\rangle .
$$

The symbol $S_{\left\{x_{a}\right\}}(a=1, \ldots, n)$ denotes the symmetrization over all $x_{a}$, which is needed because the field operators do not commute for nonequal times. The wave function $\psi$ satisfies $n$ KleinGordon equations

$$
\left(\partial_{a}^{\mu} \partial_{a \mu}+m^{2}\right) \psi\left(x_{1}, \ldots, x_{n}\right)=0
$$

one for each $x_{a}$. Although the operator $\hat{\phi}$ is hermitian, the nondiagonal matrix element $\psi$ defined by (3) is complex. Therefore, one can introduce $n$ real 4 -currents

$$
j_{a}^{\mu}=i \psi^{*} \stackrel{\leftrightarrow}{\partial_{a}^{\mu}} \psi
$$

each of which is separately conserved:

$$
\partial_{a}^{\mu} j_{a \mu}=0
$$


Equation (4) also implies

$$
\left(\sum_{a} \partial_{a}^{\mu} \partial_{a \mu}+n m^{2}\right) \psi\left(x_{1}, \ldots, x_{n}\right)=0
$$

while (6) implies

$$
\sum_{a} \partial_{a}^{\mu} j_{a \mu}=0
$$

Next we write $\psi=R e^{i S}$, where $R$ and $S$ are real functions. Equation (7) is then equivalent to a set of two real equations

$$
\begin{gathered}
\sum_{a} \partial_{a}^{\mu}\left(R^{2} \partial_{a \mu} S\right)=0 \\
-\frac{\sum_{a}\left(\partial_{a}^{\mu} S\right)\left(\partial_{a \mu} S\right)}{2 m}+\frac{n m}{2}+Q=0
\end{gathered}
$$

where

$$
Q=\frac{1}{2 m} \frac{\sum_{a} \partial_{a}^{\mu} \partial_{a \mu} R}{R}
$$

is the quantum potential. Eq. (9) is equivalent to (8), while (10) is the quantum analog of the relativistic Hamilton-Jacobi equation for $n$ particles. The corresponding classical HamiltonJacobi equation takes the same form as (10), except for the fact that there is no $Q$ term in the classical case. The Bohmian interpretation consists in postulating the existence of particle trajectories $x_{a}^{\mu}(s)$, satisfying

$$
\frac{d x_{a}^{\mu}}{d s}=-\frac{1}{m} \partial_{a}^{\mu} S
$$

Here $s$ is an affine parameter along the $n$ curves in the 4-dimensional Minkowski spacetime. These $n$ curves can also be viewed as one curve in a $4 n$-dimensional configuration space. Equation (12) has a form identical to that of the corresponding classical relativistic equation. Equation (12) can also be written in another form, as

$$
\frac{d x_{a}^{\mu}}{d s}=\frac{j_{a}^{\mu}}{2 m \psi^{*} \psi}
$$

From (12), (10), and the identity

$$
\frac{d}{d s}=\sum_{a} \frac{d x_{a}^{\mu}}{d s} \partial_{a \mu}
$$

one also finds the equation of motion

$$
m \frac{d^{2} x_{a}^{\mu}}{d s^{2}}=\partial_{a}^{\mu} Q
$$

Note that the equations above for the particle trajectories are nonlocal, but still Lorentz covariant. The Lorentz covariance is a consequence of the fact that the trajectories in spacetime do not depend on the choice of the affine parameter $s$ [14. Instead, by choosing $n$ "initial" spacetime positions $x_{a}$, the $n$ trajectories are uniquelly determined by the vector fields $j_{a}^{\mu}$ or $-\partial_{a}^{\mu} S$. More precisely, the trajectories are integral curves of these vector fields. (These two vector fields are equal up to a local positive definite scalar factor, which implies that they generate the same integral curves.) The nonlocality is encoded in the fact that the right-hand side of (15) depends not only on $x_{a}$, but also on all other $x_{a^{\prime}}$. This is a consequence of the fact that $Q\left(x_{1}, \ldots, x_{n}\right)$ in (11) is not of the form $\sum_{a} Q_{a}\left(x_{a}\right)$, which is also closely related to the fact that $S\left(x_{1}, \ldots, x_{n}\right)$ is not of the form $\sum_{a} S_{a}\left(x_{a}\right)$. The quantities $Q$ and $S$ take these forms only 
when the wave function is a product of the form $\psi\left(x_{1}, \ldots, x_{n}\right)=\psi\left(x_{1}\right) \cdots \psi\left(x_{n}\right)$ (recall that the total wave function must be symmetric for bosons). In this case there is no entanglement and the nonlocality disappears.

Note also that the fact that we parametrize all trajectories with the same parameter $s$ is not directly related to the nonlocality, because such a parametrization can be used even in local classical physics. Indeed, in a canonical approach with a single classical Hamilton-Jacobi equation that describes all $4 n$ degrees of freedom, such a parametrization is the most natural. Conversely, even in nonlocal quantum physics, once the $n$ curves $x_{a}^{\mu}(s)$ are found, one can reparametrize each of the curves with its own parameter $s_{a}$. However, when the interactions are local, then one can even use another parameter for each of the $n$ particle equations of motion themselves. For example, one can replace (12) with $d x_{a}^{\mu} / d s_{a}=-\partial_{a}^{\mu} S_{a} / m$. On the other hand, when the interactions are not local, then one must use a single parameter $s$ in the equations of motion for the trajectories, whereas new separate parameters $s_{a}$ can be used only after these equations of motion have been solved.

Now consider the nonrelativistic limit. In this limit, all wave-function frequencies are (approximately) equal to $m$, so from (15) one finds that all time components of the currents are equal and given by $j_{a}^{0}=2 m \psi^{*} \psi \equiv \tilde{\rho}$, which does not depend on $a$. Therefore, in the nonrelativistic limit, the time components of the currents become positive definite and unique for all particles. By introducing the quantity

$$
\rho\left(\mathbf{x}_{1}, \ldots, \mathbf{x}_{n}, t\right)=\left.\tilde{\rho}\left(x_{1}, \ldots, x_{n}\right)\right|_{t_{1}=\cdots=t_{n}=t},
$$

one finds that the nonrelativistic limit of (8) can be written as

$$
\frac{\partial \rho}{\partial t}+\sum_{a} \partial_{a}^{i} j_{a i}=0
$$

where $i=1,2,3$ label space coordinates. Equation (17) implies that $\rho$ can be interpreted as the probability density, which explains why the nonrelativistic limit leads to statistical transparency, but not to complete statistical transparency.

In general, in the full relativistic case, there is no analog of the function $\rho$ that would be both positive definite and independent of $a$. Such a quantity exists only in some special cases. For example, the independence of $j_{a}^{0}$ on $a$ occurs when the wave function is a product $\psi\left(x_{1}, \ldots, x_{n}\right)=\psi\left(x_{1}\right) \cdots \psi\left(x_{n}\right)$, while the positivity of $j_{a}^{0}$ occurs when $\psi(x)$ is a plane wave (not a superposition of plane waves) $\psi(x)=e^{-i p_{\mu} x^{\mu}}$ with a positive frequency $p_{0}$. In such special cases, one can introduce the quantity $\rho$ in a way similar to that of the nonrelativistic limit, leading to an equation of the form (17) valid also in the relativistic case.

Note also that the 4-currents $j_{a}^{\mu}$ do not need to be timelike, but locally can be lightlike or even spacelike. This implies that massive particles may move with the velocity of light or even faster. As shown in Ref. [11, such superluminal velocities cannot be observed, so there is no contradiction with experiments. (To understand why these superluminal velocities cannot be observed, one has to take into account the theory of quantum measurements. To emphasize the role of measurements, we also note that, contrary to frequent claims, the theory of quantum measurements is crucial for understanding why nonrelativistic Bohmian mechanics is consistent not only with the conventional statistical predictions for particle positions, but also with all statistical predictions of the conventional interpretation [3, 4, 17, 11].)

\section{TOWARDS STATISTICAL PREDICTIONS}

In order to understand more closely how interesting statistical predictions may be obtained from relativistic Bohmian mechanics, we study the one-particle case described by a wave function 
$\psi(x)$. The corresponding current is $j^{\mu}(x)$, for which we assume regularity at all $x$. In this case, one can make statitistical predictions without calculating all trajectories, provided that certain additional assumptions are fulfilled.

Let $n_{\mu}$ be the unit future-oriented timelike vector normal to an initial 3-dimensional spacelike hypersurface $\Sigma_{0}$. Assume that the quantity

$$
j=j^{\mu} n_{\mu}
$$

has the property $j \geq 0$ everywhere on $\Sigma_{0}$. Assume also that the statistical distribution of particle positions on $\Sigma_{0}$ is given by $j$. We take the normalization such that

$$
\int_{\Sigma_{0}} d S_{\mu} j^{\mu}=\int_{\Sigma_{0}} d^{3} x \sqrt{\left|g^{(3)}\right|} j=1
$$

where $d S_{\mu}=d^{3} x \sqrt{\left|g^{(3)}\right|} n_{\mu}$ is the covariant measure of the volume on $\Sigma_{0}$ and $g^{(3)}$ is the determinant of the induced metric on $\Sigma_{0}$. The normalization above corresponds to the assumption that we know with certainty that there is one and only one pointlike particle on $\Sigma_{0}$. Let the measurement of particle positions be performed at a later spacelike hypersurface $\Sigma$. Given that the initial statistical distribution is given by $j$ on $\Sigma_{0}$, what can we conclude about the statistical distribution of particle positions on $\Sigma$ ? From the local conservation $\partial_{\mu} j^{\mu}=0$, one concludes that (19) is valid also on $\Sigma$ :

$$
\int_{\Sigma} d S_{\mu} j^{\mu}=\int_{\Sigma} d^{3} x \sqrt{\left|g^{(3)}\right|} j=1
$$

where $j$ is defined by (18) with respect to an analogous normal $n_{\mu}$ on $\Sigma$. If $j \geq 0$ on $\Sigma$, then the statistical distribution on $\Sigma$ will be given by $j$. In this case, we have statistical transparency on $\Sigma$. However, a more interesting question is what if $j<0$ on some regions of $\Sigma$ ? From (20) it is clear that the inequality $j<0$ cannot be valid everywhere on $\Sigma$. Let $\Sigma^{-}$be the set of all points on $\Sigma$ at which $j<0$. For every point on $\Sigma^{-}$there exists a unique point on $\Sigma-\Sigma^{-}$, such that these two points are connected with a trajectory in $\mathcal{M}$ (where $\mathcal{M}$ is the region of Minkowski spacetime bounded by $\Sigma_{0}$ and $\Sigma$ ). Let $\Sigma^{+}$be the set of all such points on $\Sigma-\Sigma^{-}$that are connected with a point on $\Sigma^{-}$. Finally, let

$$
\Sigma^{\prime}=\Sigma-\left(\Sigma^{+} \cup \Sigma^{-}\right) .
$$

All this is illustrated in Fig. 10 The point $C^{-}$is an element of $\Sigma^{-}$, the point $C^{+}$is an element of $\Sigma^{+}$, while the points $A^{\prime}$ and $B^{\prime}$ are elements of $\Sigma^{\prime}$. The system is described by the wave function $\psi(x)$ on $\mathcal{M}$, i.e., between $\Sigma_{0}$ and $\Sigma$. The arrows on the integral curves in $\mathcal{M}$ indicate the direction of $j^{\mu}$. The dotted curves above $\Sigma$ indicate the particle trajectories that might be realized if there were no measurement of particle positions on $\Sigma$, i.e., if the system were described by $\psi(x)$ even above $\Sigma$. If there were no measurement on $\Sigma$, and if the initial position of the particle were the point $A$ on Fig. 1, then the particle would cross $\Sigma$ at 3 points, i.e., at $A^{\prime}, C^{-}$, and $C^{+}$. However, owing to the measurement of particle positions, the actual wave function above $\Sigma$ is of the form $\psi(x, y)$, where $y$ represents the degrees of freedom of the measuring apparatus. The interactions with the measuring apparatus are such that the particles enter localized channels [3, 4, 7, 11] which typically forbid trajectories such as the trajectory connecting $A^{\prime}$ with $C^{-}$. This is how the theory of quantum measurements explains why the Bohmian motions backwards in time are not in contradiction with the fact that we do not observe multiple copies of particles, such as $A^{\prime}, C^{-}$, and $C^{+}$(see also [11]). Therefore, in the rest of the discussion we ignore the dotted trajectories. Since we assume that the initial distribution is given by $j$ on $\Sigma_{0}$ (recall (19)) and its 


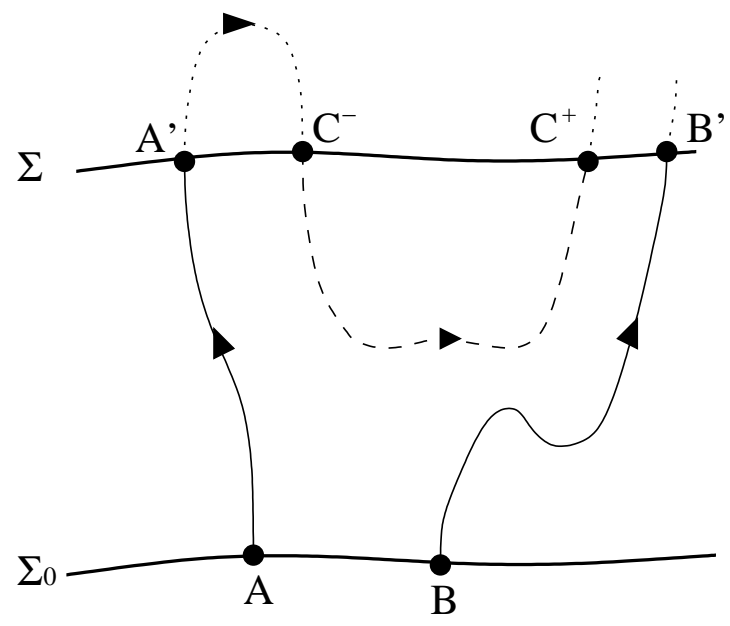

Figure 1: A sample of typical relativistic Bohmian trajectories. The solid ones represent physically realizable trajectories. The dotted ones are unphysical because the measurement takes place at the hypersuface $\Sigma$ and above. The dashed one is unphysical because it is assumed that only one particle exists and that this particle crosses the initial hypersurface $\Sigma_{0}$. The arrows indicate the direction of $j^{\mu}$.

interpretation), the dashed trajectory connecting $C^{-}$with $C^{+}$cannot be realized as an actual particle trajectory either. Only the solid trajectories can be realized as the actual trajectories.

In order to find the probability distribution of particle positions on $\Sigma$, first recall that $\Sigma$ can be decomposed in 3 disjunct sets as

$$
\Sigma=\Sigma^{\prime} \cup \Sigma^{+} \cup \Sigma^{-}
$$

Thus the integral (20) has contributions from 3 regions. These contributions have the properties

$$
\begin{gathered}
\int_{\Sigma^{\prime}} d S_{\mu} j^{\mu}=1 \\
\int_{\Sigma^{+}} d S_{\mu} j^{\mu}+\int_{\Sigma^{-}} d S_{\mu} j^{\mu}=0 .
\end{gathered}
$$

Second, note that a trajectory in $\mathcal{M}$ crosses $\Sigma^{\prime}$ if and only if it crosses $\Sigma_{0}$. (See Fig. 11 recall the definition of $\Sigma^{\prime}$ and the fact that $j \geq 0$ on $\Sigma_{0}$, and note that the integral curves of $j^{\mu}$ define a nonsingular congruence on $\mathcal{M}$.) Having this in mind and recalling (23) and the local conservation law $\partial_{\mu} j^{\mu}(x)=0$ valid on $\mathcal{M}$, it becomes evident that the probability distribution $\rho(\mathbf{x})$ on $\Sigma$ is given by

$$
\rho= \begin{cases}j & \text { on } \Sigma^{\prime} \\ 0 & \text { on } \Sigma^{+} \cup \Sigma^{-} .\end{cases}
$$

This is the measurable probability distribution. The remarkable result emerging from the existence of particle trajectories is that the probabilty for a particle to be found on the region $\Sigma^{+} \cup \Sigma^{-}$vanishes, despite the fact that $j$ does not vanish there. This is valid even if the initial distribution on $\Sigma_{0}$ is not given by $j$, only provided that we know with certainty that the particle has some (unknown) position on $\Sigma_{0}$. Then the particle cannot be found on $\Sigma^{+} \cup \Sigma^{-}$ simply because there is no trajectory in $\mathcal{M}$ connecting $\Sigma_{0}$ with $\Sigma^{+} \cup \Sigma^{-}$. The prediction that the particle cannot be found on $\Sigma^{+} \cup \Sigma^{-}$could be tested experimentally and the experimental 
confirmation of such a prediction would be a strong support for the claim that particles really do have trajectories given by the integral curves of $j^{\mu}$.

It is also interesting to note that in order to find the distribution (25), the only thing that cannot be found without the explicit calculation of the trajectories is the region $\Sigma^{+}$. Therefore, one does not need to calculate the physically realizable trajectories (the solid ones in Fig. 1), but only the unphysical ones (a dashed one in Fig. 11) connecting points in $\Sigma^{-}$with points in $\Sigma^{+}$.

Of course, in order to give a more concrete proposal for an experiment that could verify the validity of the relativistic Bohmian interpretation, one has to make a more detailed and realistic quantitative analysis of some specific case (probably with spin) in which the predictions of the Bohmian interpretation are sufficiently different from those of some other interpretation. Such a specific analysis is beyond the scope of this paper, but we hope that the presented ideas will motivate a more extensive research towards a possible experimental verification of the relativistic Bohmian mechanics.

\section{CONCLUSION}

The Klein-Gordon equation is not statistically transparent, i.e., it is not clear how to calculate the probabilities of particle positions from the knowledge of the wave function. The Bohmian interpretation, in which the probabilites only play a secondary role, provides a viable interpretation of the Klein-Gordon equation. It turns out that the lack of statistical tranparency is not a drawback but rather a virtue of the Bohmian interpretation, because it opens the possibility of experimentally distinguishing its predictions from the predictions of other possible interpretations. Another remarkable result is that the equations for Bohmian particle trajectories are

nonlocal, but they can still be naturally written in a Lorentz-covariant form without a preferred Lorentz frame.

Acknowledgements. This work was supported by the Ministry of Science and Technology of the Republic of Croatia under Contract No. 0098002.

\section{References}

[1] J. D. Bjorken and S. D. Drell, Relativistic Quantum Mechanics (McGraw-Hill, New York, 1964).

[2] J. D. Bjorken and S. D. Drell, Relativistic Quantum Fields (McGraw-Hill, New York, 1965).

[3] D. Bohm, Phys. Rev. 85, 166, 180 (1952).

[4] D. Bohm and B. J. Hiley, Phys. Rep. 144, 323 (1987).

[5] D. Bohm, B. J. Hiley, and P. N. Kaloyerou, Phys. Rep. 144, 349 (1987).

[6] P. R. Holland, Phys. Rep. 224, 95 (1993).

[7] P. R. Holland, The Quantum Theory of Motion (Cambridge University Press, Cambridge, 1993).

[8] H. Nikolić, Phys. Lett. B 527, 119 (2002); Erratum 529, 265 (2002).

[9] H. Nikolić, Int. J. Mod. Phys. D 12, 407 (2003). 
[10] H. Nikolić, hep-th/0205022, to appear in Gen. Rel. Grav.

[11] H. Nikolić, Found. Phys. Lett. 17, 363 (2004).

[12] H. Nikolić, quant-ph/0302152, to appear in Found. Phys. Lett.

[13] H. Nikolić, quant-ph/0307179.

[14] K. Berndl, D. Dürr, S. Goldstein, and N. Zanghì, Phys. Rev. A 53, 2062 (1996).

[15] S. Liberati, S. Sonego, and M. Visser, Ann. Phys. 298, 167 (2002).

[16] H. Nikolić, gr-qc/0403121.

[17] C. G. B. Garrett and D. E. McCumber, 1970 Phys. Rev. A 1, 305 (1970).

[18] S. Chu and S. Wong, Phys. Rev. Lett. 48, 738 (1982).

[19] I. T. Drummond and S. J. Hathrell, Phys. Rev. D 22, 343 (1980).

[20] K. Scharnhorst, Phys. Lett. B 22, 354 (1990).

[21] T. Y. Chiao, Phys. Rev. A 48, R34 (1993).

[22] E. Bolda, R. Y. Chiao, and J. C. Garrison, Phys. Rev. A 48, 3890 (1993).

[23] R. Y. Chiao, A. E. Kozhekin, and G. Kurizki, Phys. Rev. Lett. 77, 1254 (1996).

[24] N. Bilić and H. Nikolić, Phys. Rev. D 68, 085008 (2003).

[25] D. Dürr, S. Goldstein, K. Münch-Berndl, and N. Zanghì, Phys. Rev. A 60, 2729 (1999).

[26] K. Kuchař, in: Proceedings of the 4th Canadian Conference on General Relativity and Relativistic Astrophysics (World Scientific, Singapore, 1992).

[27] C. J. Isham, gr-qc/9210011.

[28] P. Holland, Phys. Rev. A 60, 4326 (1999).

[29] P. R. Holland and J. P. Vigier, Nuovo Cimento 88B, 20 (1985).

[30] C. L. Lopreore and R. E. Wyatt, Phys. Rev. Lett. 82, 5190 (1999).

[31] D. Dürr, S. Goldstein, and N. Zanghì, J. Stat. Phys. 67, 843 (1992).

[32] D. Dürr, S. Goldstein, and N. Zanghì, Phys. Lett. A 172, 6 (1992).

[33] A. Valentini, Phys. Lett. A 156, 5 (1991).

[34] S. S. Schweber, An Introduction to Relativistic Quantum Field Theory (Harper \& Row, New York, 1961). 